\title{
REDE DE FRIO PARA OS PRINCIPAIS IMUNOBIOLÓGICOS ADOTADOS NO BRASIL: ESTAMOS PREPARADOS?
}

Mayra Martho Moura de Oliveira'," ORCID: 0000-0002-6965-9204

Evelin Placido dos Santos"'I ORCID: 0000-0002-6152-0072

Josineia Leite de Oliveiralv ORCID: 0000-0001-7023-541X

'Sociedade Brasileira de Imunizações - SBIm. São Paulo, SP.

"Instituto Butantan. São Paulo, SP.

I'Projeto Xingu da Universidade Federal de São Paulo. São Paulo, SP.

"Secretaria de Estado da Saúde de São Paulo. São Paulo, SP.

Autora Correspondente: Mayra Martho Moura de Oliveira E-mail:mayra@capacitaimune.com.br

\section{INTRODUÇÃO}

A rede de frio é o sistema técnico-administrativo responsável pela manutenção da cadeia de frio, que é processo logístico de conservação dos imunobiológicos desde a produção até a administração no usuário, passando pelas etapas de recebimento, inspeção, armazenamento, separação, distribuição e transporte, garantindo assim a qualidade, eficácia e preservando suas características. Os imunobiológicos são produtos termolábeis (sensíveis ao calor e ao frio) e fotossensíveis (sensíveis à luz), sendo que o monitoramento e controle de temperatura são determinantes para o sucesso da operacionalização da cadeia de frio. No Brasil existe uma grande diversidade de imunobiológicos, com temperaturas de armazenamento diferentes, inclusive em cada instância (central, regional e local), o que torna esse processo delicado exigindo atenção e monitoramento contínuo ${ }^{(1)}$.

\section{CARACTERÍSTICAS E ARMAZENAMENTO DOS IMUNOBIOLÓGICOS}

Cada imunobiológico possui características específicas e para sua manutenção o laboratório produtor define qual é a condição ideal para o armazenamento em cada instância, conforme sua composição e forma farmacêutica (líquida ou liofilizada).Sua apresentação pode ser unidose de uso imediato ou multidose, neste caso também é definido pelo laboratório produtor a validade e a temperatura de conservação(2).

O Instituto Nacional de Controle de Qualidade em Saúde - INCQS tem a responsabilidade de liberação de todos os lotes dos imunobiológicos utilizados no Brasil, tanto no segmento público, quanto no privado(1-4).

A conservação dos imunobiológicos deve ter controle de temperatura rigoroso e o tempo de armazenamento deve seguir as orientações da Organização Mundial da Saúde (OMS) que foram adotadas pelo Programa Nacional de Imunizações - PNI ${ }^{(1-2,11)}$. 
Quadro 1 - Informações sobre conservação, temperatura, local e tempo de armazenamento, segundo imunobiológico e instâncias central e regional. Brasil, $2021^{(11)}$

\begin{tabular}{|c|c|c|c|}
\hline $\begin{array}{c}\text { Local e tempo de } \\
\text { armazenamento } \\
\text { Temperatura }\end{array}$ & $\begin{array}{c}\text { Central } \\
\text { Nacional/Estadual } \\
6 \text { a } 12 \text { meses }\end{array}$ & $\begin{array}{c}\text { Regional } \\
\text { Regional/Municipal/CRIE } \\
3 \text { a } 6 \text { meses }\end{array}$ & $\begin{array}{l}\text { Local } \\
\text { Sala de Vacinação }\end{array}$ \\
\hline$-90^{\circ} \mathrm{C} \mathrm{a}-60^{\circ} \mathrm{C}$ & COVID/Pfizer/Comirnaty* & COVID/Pfizer/Comirnaty* & $\begin{array}{c}+2^{\circ} \mathrm{a}+8^{\circ} \\
1 \text { mês }\end{array}$ \\
\hline$-25^{\circ} \mathrm{Ca}-15^{\circ} \mathrm{C}$ & $\begin{array}{l}\text { COVID/Pfizer/Comirnaty* } \\
\text { Pólio Oral } \\
\text { Febre Amarela/ Fiocruz }\end{array}$ & & $+2^{\circ} \mathrm{a}+8^{\circ}$ \\
\hline$+2^{\circ} \mathrm{Ca}+8^{\circ} \mathrm{C}$ & $\begin{array}{l}\text { Imunoglobulinas, Soros, } \\
\text { demais vacinas }\end{array}$ & $\begin{array}{l}\text { Imunoglobulinas, Soros, } \\
\text { demais vacinas Diluentes**** }\end{array}$ & $\begin{array}{l}\text { Imunoglobulinas, Soros, } \\
\text { Todas as vacinas e diluentes }\end{array}$ \\
\hline Ambiente até $25^{\circ} \mathrm{C}$ & Diluentes $* * * *$ & Diluentes $* * * *$ & \\
\hline \multicolumn{4}{|c|}{ Fonte: Informaçōes Globais sobre COMIRNATY(11). (Adaptado pelo autor). } \\
\hline \multicolumn{4}{|c|}{ 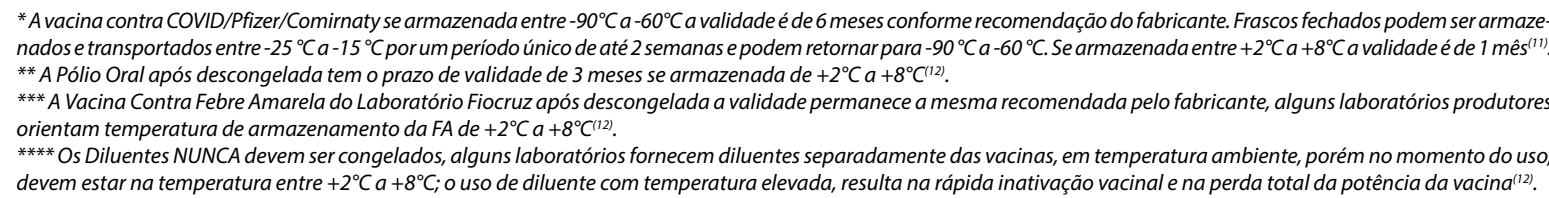 } \\
\hline
\end{tabular}

\section{MONITORAMENTO DE TEMPERATURA E NOTIFICAÇÃO DE ALTERAÇÃO}

O monitoramento de temperatura deve ser rigoroso em todas as instâncias para garantir a qualidade dos imunobiológicos. Atualmente existem diversas formas de registro de temperatura que podem ser eletrônicas ou manuais ${ }^{(1)}$.

Os registros do monitoramento devem ser armazenados e guardados, por, pelo menos, dois anos após sua geração(3).

Alteração de temperatura ou queixa técnica relacionada ao serviço de vacinação deve ser comunicada à Divisão de Imunização Estadual, e a notificação deve ser realizada no Sistema de Notificações em Vigilância Sanitária - Notivisa ${ }^{(4-5)}$.

Para notificação de alteração de temperatura ou queixa técnica da vacina contra COVID-19, a Equipe Técnica da Rede de Frio e Controle de Qualidade/PNI desenvolveu um formulário a fim de facilitar e agilizar a comunicação de ocorrências e orientar as ações preventivas ${ }^{(5-8)}$.

Após acessar o formulário, é necessário escolher a opção "Temperatura de conservação diferente da definida na bula" no tópico 2 "Desvio de Qualidade". Desta forma, será possível preencher os campos referentes à alteração de temperatura ${ }^{(5-11)}$.

Os registros e formulários serão submetidos ao PNI para avaliação do Instituto Nacional de Controle de Qualidade em Saúde (INCQS). Durante o processo de comunicação e análise, o lote do imunobiológico exposto deve ser segregado, identificado e preservado à temperatura adequada de $+2^{\circ} \mathrm{Ca}+8^{\circ} \mathrm{C}^{(5)}$.

\section{BOAS PRÁTICAS DE ARMAZENAMENTO E DISTRIBUIÇÃO DE IMUNOBIOLÓGICOS}

É por meio de padronização dos procedimentos e práticas apropriadas conforme legislação vigente, que será garantida a conservação da potência imunogênica dos imunobiológicos conferida pelo laboratório 
produtor. Todos os procedimentos de recebimento, armazenamento, expedição, distribuição e transporte devem estar descritos em Procedimentos Operacionais Padrão (POP), atualizados periodicamente e acessíveis aos profissionais que atuam no processo ${ }^{(1-3)}$.

\section{CUIDADOS NO RECEBIMENTO E INSPEÇÃO DOS IMUNOBIOLÓGICOS:}

- Verificar o tipo de embalagem, as condições de transporte e armazenagem aplicáveis, se a embalagem está lacrada e identificada(1);

- Verificar a integridade da carga ${ }^{(1)}$;

- Verificar a temperatura dos volumes recebidos(1);

- Conferir os números de lote, data de validade e quantidades recebidas, se estão de acordo com os pedidos efetuados e notas fiscais recebidas ${ }^{(1)}$;

- O tempo de inspeção deve ser o mais breve possível a fim de minimizar o tempo de exposição do imunobiológico fora do recomendado pelo laboratório produtor ${ }^{(1)}$;

- Após a inspeção os imunobiológicos devem ser armazenados conforme recomendação do fabricante ${ }^{(1)}$;

- Os imunobiológicos fora do padrão recomendado devem ser devolvidos no ato do recebimento ou devem ser identificados e colocados em quarentena enquanto aguardam a resposta da notificação(1).

Deve haver um número adequado de profissionais treinados para garantir que as responsabilidades atribuídas individualmente não apresentem riscos à qualidade do produto. $O$ treinamento deve ser aplicado no ingresso do profissional e deve permanecer de forma contínua, proporcional a complexidade da função ${ }^{(3)}$.

\section{ARMAZENAMENTO DE IMUNOBIOLÓGICOS}

As câmaras refrigeradas são aplicáveis para o armazenamento dos imunobiológicos em temperatura positiva, de $2^{\circ} \mathrm{C}$ a $8^{\circ} \mathrm{C}^{(1)}$.

Os freezers são indicados para o armazenamento de imunobiológicos em temperatura negativa, $-25^{\circ} \mathrm{C}$ a $-15^{\circ} \mathrm{C}$, e para o armazenamento das bobinas reutilizáveis necessárias à conservação dos imunobiológicos em caixas térmicas para transporte e/ou procedimentos nas salas de imunização(1).

\section{CUIDADOS PARA UTILIZAÇÃO DOS EQUIPAMENTOS}

- Usar tomada ou conexão com a fonte de energia elétrica, exclusivos para a câmara(1);

- Colocar a câmara distante da fonte de calor, nivelado e afastado da parede, pelo menos $20 \mathrm{~cm}^{(1)}$;

- Usar câmara única e exclusivamente para o armazenamento dos imunobiológicos ${ }^{(1)}$;

- Identificar o equipamento de maneira visível ${ }^{(1)}$;

- Manter termostato regulado para temperatura entre $+2^{\circ} \mathrm{Ce}+8^{\circ} \mathrm{C}$, temperatura média $+5^{\circ} \mathrm{C}^{(1)}$;

- Elaborar "Mapa llustrativo" com a posição de cada vacina no interior do equipamento (1);

- Manter sistema de alarme ou geradores elétricos de emergência ${ }^{(1)}$;

- Utilizar termômetro para leitura de máxima e mínima ${ }^{(1)}$;

- Fazer a leitura do termômetro e registro da temperatura no mínimo duas vezes ao dia, ou idealmente três vezes ${ }^{(1)}$;

- Organizar as vacinas próximas do vencimento na frente das prateleiras da câmara, sem a necessidade de diferenciar por tipo, uma vez que as câmaras refrigeradas possuem distribuição uniforme de temperatura no seu interior ${ }^{(1)}$. 


\section{EQUIPAMENTOS UTILIZADOS PARA MEDIÇÃO DE TEMPERATURA}

- Os termômetros Max./Min.com cabo extensor, termômetro a laser, termômetro tipo espeto e datalogger são os equipamentos mais utilizados para medição de temperatura na rede de frio ${ }^{(1)}$.

- O Datalogger é o equipamento recomendado para utilização no monitoramento de temperatura no transporte de imunobiológicos, pois registra a temperatura durante todo percurso permitindo avaliação posterior de possível alteração de temperatura ${ }^{(1)}$.

Equipamentos recomendados para sala de vacina e armazenamento para manutenção da rede de frio.

- Condicionador de ar para climatização dos ambientes, de manipulação e preparo para aplicação(1).

- Grupo gerador de energia aplicado às situações emergenciais para suprimento de energia elétrica ${ }^{(1)}$.

- Termômetros digitais e a lazer: troca de baterias de 6/6 meses, anotando data de troca e calibração anual, por laboratório credenciado pela RBC/INMETRO (1).

- Registrador eletrônico de temperatura (Logger): calibração anual, por laboratório credenciado pela RBC/INMETRO (1).

- Câmaras: qualificação anual por laboratório credenciado pela RBC/INMETRO(5).

Todos os equipamentos utilizados na rede de frio devem ser adquiridos mantendo critérios de seleção recomendados pelo Manual de Rede de Frio do Ministério da Saúde e legislação vigente, devendo ser submetidos periodicamente aos procedimentos de manutenção e calibração(1).

\section{VACINAÇÃO EXTRAMUROS}

É a atividade vinculada a um serviço de vacinação licenciado, que ocorre de forma esporádica, fora do estabelecimento de vacinação (Quadro 2$)^{(9)}$.

Quadro 2 - Equipamentos e insumos da cadeia de frio necessários para as atividades de vacinação extramuros. Brasil, 2017(1)

\begin{tabular}{|c|c|}
\hline \multicolumn{2}{|c|}{ Materiais necessários } \\
\hline Caixa térmica com termômetro Max. E Min. para estoque & Caixa térmica com termômetro Max . E Min. Para aplicação \\
\hline Caixa térmica com bobinas de gelo & Álcool líquido e em gel $70^{\circ} \%$ \\
\hline Bandeja de inox & Algodão \\
\hline Seringas e agulhas & Curativo \\
\hline Comprovante de vacinação & Folha de monitoramento de temperatura \\
\hline Saco de Lixo infectante e comum & Coletor de perfuro cortante \\
\hline
\end{tabular}

Fonte: Manual de Rede de Frio do Programa Nacional de Imunizações ${ }^{(1)}$. (Adaptado pelo autor)

Para a vacinação extramuros o registro da temperatura das caixas térmicas deve ser aferido a cada hora, durante todo o tempo que as vacinas permanecerem fora do equipamento específico de armazenamento das vacinas ${ }^{(1-9)}$. 


\section{PREPARO DA CAIXA TÉRMICA}

- Manter as bobinas em congelador durante um período mínimo de 48 h;

- Estar atento a validade das bobinas

- Retirar as bobinas do freezer;

- Colocar as bobinas sobre a superfície até desaparecer a névoa, simultaneamente monitorar a temperatura de uma delas com termômetro de cabo extensor (temperatura de $0^{\circ} \mathrm{C}$ );

- Secar e colocar as bobinas na caixa térmica;

- Estabelecer a quantidade adequada de bobinas para a quantidade de imunobiológicos;

- Dispor as bobinas no fundo e nas paredes internas;

- Utilizar barreiras (plástico bolha, papelão, berço) entre as vacinas e as bobinas;

- Estabilizar a temperatura interna da caixa, (intervalo de $+2^{\circ} \mathrm{C}$ a $+8^{\circ} \mathrm{C}$, ideal $+5^{\circ} \mathrm{C}$ ) antes de colocar as vacinas;

- Organizar os imunobiológicos no interior da caixa de maneira segura para que não fiquem soltos e, eventualmente, desloquem-se sofrendo impactos mecânicos durante o transporte;

- Colocar o datalogger e/ou bulbo do termômetro no centro da caixa, em contato somente com o frasco da vacina, afixando o termômetro no exterior da caixa;

- Dispor as bobinas reutilizáveis cobrindo os imunobiológicos, sendo utilizadas como barreira entre as vacinas;

- Fechar a caixa térmica, sem deixar frestas, se necessário, usar fita adesiva;

- Monitorar a temperatura durante todo o processo até a entrega;

- Identificar as caixas.

Para as vacinas congeladas: as caixas são montadas com gelo seco ou bobinas congeladas ${ }^{(11)}$.

\section{CUIDADOS NO TRANSPORTE DE IMUNOBIOLÓGICOS}

Os imunobiológicos são produtos termolábeis (sensíveis ao calor e ao frio) e fotossensíveis (sensíveis à luz). Assim, devem ser armazenados, transportados, organizados, monitorados, distribuídos e administrados adequadamente, de forma a manter sua eficácia e potência, ou seja, sua capacidade de resposta. A potência é um dos fatores que interfere na magnitude e na duração da resposta imune ${ }^{(1)}$.

Recomenda-se o uso de veículo refrigerado e, nos percursos mais longos, orienta-se utilizar caixa térmica exclusivamente para o transporte de bobinas reutilizáveis congeladas para efetuar a troca durante o trajeto, quando necessário(1).

Na impossibilidade da utilização de veículos refrigerados é indispensável o uso de veículo climatizado, posicionando as caixas térmicas distantes de fontes de calor e protegidas da incidência de luz solar direta. Em eventuais paradas o veículo deve ser estacionado à sombra e a climatização interna deve ser mantida(1).

A vacina Comirnaty, da Pfizer tem características de armazenamento diferentes das usualmente praticadas no Brasil, devendo seguir atualmente essa orientação para rede de frio ${ }^{(11)}$ :

- Os frascos fechados podem ser armazenados e transportados entre $-25^{\circ} \mathrm{C}$ a $-15{ }^{\circ} \mathrm{C}$ por um período único de até 2 semanas e podem retornar a $-90^{\circ} \mathrm{C}$ a $-60^{\circ} \mathrm{C}$.

- Uma vez retirada do congelador, a vacina fechada pode ser armazenada por até 1 mês entre $+2^{\circ} \mathrm{Ce}+8^{\circ} \mathrm{C}$.

- Dentro do prazo de validade de 1 mês a $2^{\circ} \mathrm{C}$ a $8^{\circ} \mathrm{C}$, até 12 horas podem ser usadas para o transporte.

- Antes do uso, a vacina fechada pode ser armazenada por até 2 horas a temperatura até $30^{\circ} \mathrm{C}$.

- Uma vez descongelada, a vacina não deve ser congelada novamente. 


\section{GERENCIAMENTO DE RESÍDUOS DE VACINAÇÃO}

Recomenda-se o descarte dos resíduos da vacinação de acordo com os processos utilizados para outras vacinas. O Plano de Gerenciamento de Resíduos do Serviço de Saúde deve ser seguido pelos profissionais treinados que realizam o manejo do resíduo, de acordo com o proposto na Resolução da Diretoria Colegiada - RDC $n^{\circ} 222$, de 28 de março de 2018, que dispõe sobre o regulamento técnico para o gerenciamento de Resíduos de Serviços de Saúde e a Resolução Conama n 358, de 29 de abril de 2005, que dispõe sobre o tratamento e a disposição final dos Resíduos dos Serviços de Saúde (RSS) ${ }^{(7-8,10)}$.

O gerenciamento dos resíduos de vacinação no Brasil deve seguir as definições estabelecidas na Resolução no 18, de 23 de março de 2018, que dispõe sobre a classificação de riscos de Organismos Geneticamente Modificados (OGM) e os níveis de biossegurança a serem aplicados nas atividades e projetos com OGM e seus derivados em contenção ${ }^{(8)}$.

\section{CONCLUSÃO}

Nesse processo, devem atuar pessoas treinadas, capacitadas e responsáveis com a execução dos procedimentos, para garantir a qualidade e a eficácia do imunobiológico.

O uso de equipamentos e monitoramento adequados, conhecimento sobre conservação, transporte e manutenção da temperatura das vacinas e a preocupação com as consequências trazidas pelo congelamento dos imunobiológicos que não devem ser congelados, se faz necessário para manutenção da eficiência da rede de frio e toda a sua extensão, para garantir a qualidade das vacinas.

\section{REFERÊNCIAS}

1. Ministério da Saúde (BR). Secretaria de Vigilância em Saúde. Departamento de Vigilância das Doenças Transmissíveis. Manual de Rede de Frio do Programa Nacional de Imunizações [Internet]. 5. ed. Brasília: Ministério da Saúde; 2017[cited 2021 Jul 10]. 136 p.: il. Available from: https://bvsms.saude.gov.br/bvs/publicacoes/manual_rede_frio4ed.pdf

2. Ministério da Saúde (BR). Secretaria de Vigilância em Saúde. Departamento de Imunização e Doenças Transmissíveis. Manual dos Centros de Referência para Imunobiológicos Especiais [Internet]. 5. ed. Brasília: Ministério da Saúde; 2019 [cited 2021 Jul 10]. 174 p.: il. Available from: https://sbim.org.br/images/calendarios/manual-centros-referencia_ imunobiologicos-especiais-5ed-web.pdf

3. Ministério da Saúde (BR). Resolução - RDC № 430, de 8 de outubro de 2020. Dispõe sobre as Boas Práticas de Distribuição, Armazenagem e de Transporte de Medicamentos [Internet]. 09/10/2020[cited $2021 \mathrm{Jul} 10$ ].195(1):110. Available from: https://www.in.gov.br/en/web/dou/-/resolucao-de-diretoria-colegiada-rdc-n-430-de-8-de-outubro-de-2020-282070593

4. Ministério da Saúde (BR). Secretaria de Vigilância em Saúde. Departamento de Imunizações e Doenças Transmissíveis. Manual de vigilância epidemiológica de eventos adversos pós-vacinação [Internet]. 4. ed. Brasília: Ministério da Saúde; 2020[cited 2021 Jul 10]. Available from: https://sbim.org.br/images/files/manual-vigilanciaepidemiologica-eventos-vacinacao-4ed.pdf

5. Ministério da Saúde (BR). Secretaria de Vigilância em Saúde. Departamento de Vigilância Epidemiológica. Coordenação Geral do Programa Nacional de Imunização. Formulário para Notificação/Investigação de Eventos Adversos Pós-Vacinação associados ao uso de Vacina, Soro ou Imunoglobulina. Dez. 2010.

6. Agência Nacional de Vigilância Sanitária. Resolução no 18, de 23 de março de 2018, que dispõe sobre a classificação de riscos de Organismos Geneticamente Modificados (OGM) e os níveis de biossegurança a serem aplicados nas atividades e projetos com OGM e seus derivados em contenção [Internet]. 2018[cited 2021 Jul 10]. Available from: http://ctnbio.mctic. gov.br/resolucoes-normativas

7. Agência Nacional de Vigilância Sanitária. Resolução da Diretoria Colegiada RDC n 222, de 28 de março de 2018 , que dispõe sobre o regulamento técnico para o gerenciamento de Resíduos de Serviços de Saúde [Internet]. 2018 [cited 2021 Jul 10]. Available from: https://bvsms.saude.gov.br/bvs/saudelegis/anvisa/2018/rdc0222_28_03_2018.pdf 
8. Governo do Estado de São Paulo. Divisão de Imunização Centro de Vigilância Epidemiológica "Prof. Alexandre Vranjac" CVE. Documento Técnico Campanha de Vacinação contra a COVID-19 [Internet]. 16a atual. 2021 [cited 2021 Jul 10] Available from: https://portal.saude.sp.gov.br/resources/cve-centro-de-vigilancia-epidemiologica/vacina/documentos-tecnicoscovid-19/documentotecnico_campanhadevacinacaocontraacovid_16atualizacao_23_06.pdf

9. Agência Nacional de Vigilância Sanitária. Resolução RDC 197 Dispõe sobre os requisitos mínimos para o funcionamento dos serviços de vacinação humana [Internet]. 26 de dezembro de 2017[cited 2021 Jul 10]. Available from: https://sbim.org. br/legislacao/867-rdc-anvisa-n-197-26-de-dezembro-de-2017

10. Conselho Nacional do Meio Ambiente-CONAMA. Resolução Conama n 358, de 29 de abril de 2005, que dispõe sobre o tratamento e a disposição final dos Resíduos dos Serviços de Saúde (RSS) [Internet]. 2021 [cited 2021 Jul 10]. Available from: http://www2.mma.gov.br/port/conama/legiabre.cfm?codlegi=462

11. Pfizer. Comirnaty COVID 19 Vaccine mRNA (nucleoside modified). Informações Globais sobre Comirnaty [Internet]. 2021 [cited $2021 \mathrm{Jul}$ 10]. Available from: https://www.comirnatyeducation.com.br/

12. Ministério da Saúde (BR). Secretaria de Vigilância em Saúde. Departamento de Vigilância Epidemiológica. Manual de rede de frio. 4. ed. Brasília: Ministério da Saúde; 2013. 